\section{REDUCING THE USE OF SECLUSION: CREATING A CULTURE OF SAFETY AND TEAMWORK IN A NEW INPATIENT PSYCHIATRIC UNIT}

Nicole Powell, Jahnavi Valleru, Rajeev Krishna, Jodi Finney. Nationwide Childrens Hospital, US

\subsection{6/bmjoq-2017-IHI.16}

Background Seclusion is an intensive intervention employed in inpatient mental health units to guarantee safety when de-escalation methods have been exhausted. High rate of seclusion are associated with higher employee injury rate, lower staff engagement and increased patient length of stay.

Objectives Our aim is to reduce seclusion rates in all admitted patients in inpatient psychiatry from a baseline of 136 to less than 110 per 1000 patient days by December 2016 and sustain for 12 months.

Methods A multidisciplinary team developed an Aim and Key Driver Diagram focused on reducing seclusions. Evidence based interventions included milieu management techniques, proactive patient engagement and staff training on trauma informed approaches, physical deflection, and reinforcement principles. Other successful Plan-Do-Study-Act's (PDSA's) include small patient groups and skill-appropriate programming to reduce opportunities for escalating behaviours and increase patient success.

Results By 1st quarter 2017, seclusion rates were reduced from 136 to 60 seclusions per 1000 patient days (56\% reduction) and sustained for 12 months. In addition to seclusion rates, we achieved a $40 \%$ reduction in duration of seclusions, a $55 \%$ reduction in mechanical restraints and a $72 \%$ reduction in employee injuries.

Conclusions Seclusion in children and adolescent mental health units can be significantly reduced through systematic application of quality improvement methodology to revise unit programming and address training and awareness issues. We implemented several effective and less-disruptive interventions while we established a new unit and trained inexperienced staff. These strategies may help impact care of patients in other child and adolescent mental health units.

\section{IMPROVING TIMELY TRANSITION OF PARENTERAL TO ENTERAL ANTIBIOTICS IN PAEDIATRIC PATIENTS WITH PNEUMONIA AND CELLULITIS USING QUALITY IMPROVEMENT METHODS}

Sonya Tang Girdwood, Maria Sellas, Joshua Courter, Brianna Liberio, Michael Tchou, Lisa Herrmann, Maya Dewan, Angela Statile, Ndidi Unaka. Cincinnati Children's Hospital Medical Centre, US

\subsection{6/bmjoq-2017-IHI.17}

Background Early transition of parenteral (IV) to enteral antibiotics is recommended to decrease hospital length of stay and healthcare costs. Without clear criteria to guide transition, patients at our hospital often remained on IV antibiotics until discharge despite clinical improvement.

Objectives Increase the percentage of timely-transitioned antibiotic doses (total enteral antibiotic doses divided by all antibiotic doses in patients receiving other enteral medications) for Hospital Medicine (HM) patients admitted for uncomplicated pneumonia or skin and soft tissue infections (SSTI) from $44 \%$ to $75 \%$ by August 31, 2017.

Methods Improvement efforts targeted five HM teams at a large paediatric academic hospital. Our multidisciplinary team included HM attendings, fellows, residents, and pharmacists. Several key drivers (Figure 1) informed our interventions. Interventions included education on IV and enteral antibiotic cost differential, incorporation of antibiotic transition plan in electronic health record (EHR) note templates, structured discussions of transition criteria for patients on IV antibiotics, and real-time identification of failures and feedback. An automated system that interfaced with our EHR provided data on medication administration route and missed opportunities for timely-transitioned antibiotic doses. An annotated statistical process control chart assessed the impact of interventions over time (figure 2).

Results The percentage of timely-transitioned antibiotic doses increased from $44 \%$ to $80 \%$ within 8 months. The most effective interventions were early identification of transition criteria through structured huddles and real-time identification and mitigation of failures.

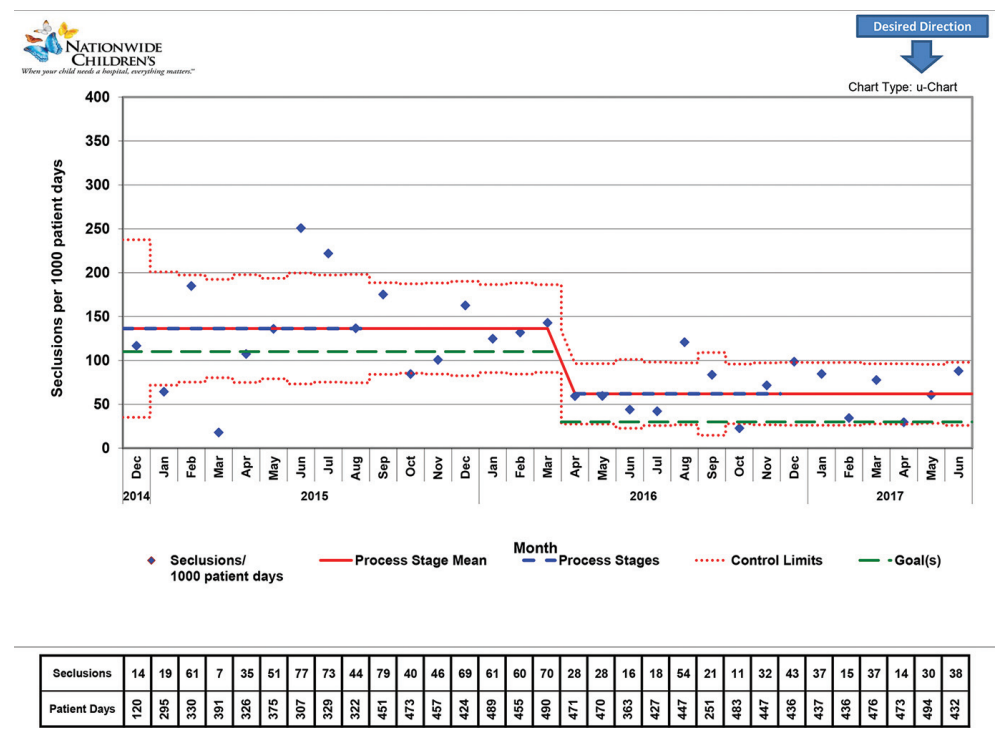

Abstract 946 Figure 1 Number of seclusions per 1000 patient days in T5A 